\title{
Review of: "Bayesian inference of multi-point macromolecular architecture mixtures at nanometre resolution"
}

\author{
Maria Ott ${ }^{1}$ \\ 1 Martin-Luther Universität Halle-Wittenberg
}

Potential competing interests: The author(s) declared that no potential competing interests exist.

This review refers to the preprint posted on Aug 22, 2021

The article addresses the important issue to retrieve not only qualitative but quantitative information from stacks of single particle images. By now, many groups are able to obtain fluorescent images with high resolution, but the conversion of such images to real positions and distances is still challenging.

The authors discuss the current problem of the overestimation of the particle to particle distances and display a simple methodological approach of image processing in combination with a Markov Chain Monte Carlo algorithm to reveal different states.

The addition of new constraints, e.g. constant sum of the inner angles of a polygon, improves the determination of the pair-wise distances compared to the pairwise Bayesian approach BEDCA. A second improvement is the implementation of an anisotropic measurement error evolving from the anisotropic detection volume of the microscopes.

Minor issues:

1) Since most of the distances, especially the larger distances between 50 and $70 \mathrm{~nm}$ agree well with former models I have two issues addressing the determination of shorter distances:

- Why is the Nnf2-Ndc80c distance of "example 4" (Table 3) obtained by the triangle approach deviating from the BEDCA approach, while there is an agreement for "example 5" and "example 3"? This is contra-intuitive since the numbers $\mathrm{N}$ and $\mathrm{C}$ of "example 4" are much larger and the other 2 distances much longer. This is also contradicting the results of Table 1.

- The simulation of the triangle of $a=15, b=15, c=15$ (Tab 1 ) shows a large deviation for one side of the triangle. Hence the accuracy of "example 5" of Table 3 can hardly be verified. Is there any other way to prove the result? Can this underestimation of the length of a single side of the triangle be explained? 
2) More general:

- As a minimum of 3 consecutive images of one state are required and the photons per particles are experimentally limited - Could you give any relation (or plot) how the number of photons per particle/number of frames relate to the uncertainty and to the min. obtainable inter-particle distances? It would be good to compare these numbers to BEDCA or alternative approaches to strengthen the improvement by the triangular approach. (The Tables and plots of the manuscript are still too complex.) This would also make the article more useful for a broader audience

- Is there a way to make the program available to other groups that are interested for this type of image analysis? 